Trauma Berufskrankh 2010 · 12:5 DOI 10.1007/s10039-010-1592-0

Online publiziert: 28. Februar 2010

(c) Springer-Verlag 2010
T. Kälicke • G. Muhr

Chirurgische Universitätsklinik und Poliklinik, BG-Universitätsklinikum Bergmannsheil, Bochum

\section{Primäre Endoprothetik nach Trauma}

Die bessere Alternative?
Das Leitthema dieses Themenhefts ist die primäre Endoprothetik nach Trauma, ein Thema, das zum einen aufgrund des demografischen Wandels, zum anderen aber auch aufgrund der Zusammenführung der Fachbereiche Unfallchirurgie und Orthopädie zunehmend in den Fokus gerät.

Die Rekonstruktion und osteosynthetische Versorgung von Gelenk- und gelenknahen Frakturen galt lange Zeit uneingeschränkt für alle Patientengruppen als Goldstandard der operativen Behandlung solcher Verletzungen. Für junge Patienten gilt dies auch heute noch nahezu ausnahmslos. Die großen Erfolge der Endoprothetik in der Behandlung degenerativer Erkrankungen insbesondere der großen Gelenke ließen aber zunehmend die Frage aufkommen, ob bestimmte Patientengruppen, insbesondere ältere Patienten mit verminderter Compliance und/ oder bestehender Unfähigkeit, eine Entlastung- bzw. Teilbelastung, wie bei der osteosynthetischen Versorgung solcher Frakturen erforderlich, durchzuführen, nicht eher von einer primären prothetischen Versorgung nach Trauma profitieren, da hier deren zentraler Vorteil die sofortige postoperative Möglichkeit zur Vollbelastung - zum Tragen kommt. Hinzu kam die nicht unbeträchtlich hohe Anzahl posttraumatischer Arthrosen nach primär osteosynthetischer Versorgung von Gelenkfrakturen mit konsekutiver Notwendigkeit der prothetischen Versorgung.

Die primäre Hemi- und Vollprothesenimplantation bei der Therapie der dislozierten medialen Schenkelhalsfraktur des alten Menschen ist nahezu überall als Verfahren der Wahl etabliert. Auch in der Schulterchirurgie hat die Endoprothese nach Trauma mittlerweile einen festen Stellenwert. Zunehmend an Bedeutung scheint auch die primär prothetische Versorgung intraartikulärer distaler Humerusfrakturen zu gewinnen, betrachtet man die derzeit drastisch ansteigende Zahl an Publikationen zu dieser Thematik. Trotz ausgereifter und vielfach bewährter Endoprothesenmodelle hat im Gegensatz hierzu die primäre Endoprothetik nach komplexer kniegelenknaher Fraktur bzw. intraartikulärem Bruch des distalen Femurs bzw. der proximalen Tibia weiterhin nahezu keine Bedeutung.

Die Abgrenzung, wann welches Verfahren für welchen Patienten in der jeweiligen Situation tatsächlich geeignet ist und angewendet werden sollte, ist oft schwierig und wird weiterhin lebhaft kontrovers diskutiert. Vieles ist hierbei leider eher gefühlt, wenig evidenzbasiert. Der vorliegende Band der Zeitschrift Trauma und Berufskrankheit spiegelt den derzeitigen Stellenwert der primären Endoprothetik nach Trauma unter kritischer Beurteilung der vorhandenen Literatur wider.

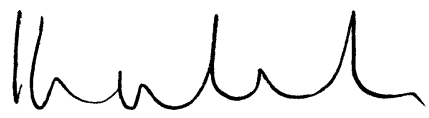

T. Kälicke

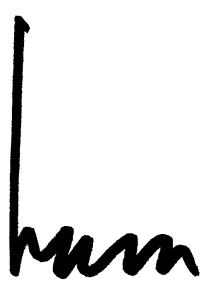

G. Muhr

\section{Korrespondenzadresse}

PD Dr. T. Kälicke

Chirurgische Universitätsklinik und Poliklinik, BG-Universitätsklinikum Bergmannsheil, Bürkle-de-la-Camp-Platz 1, 44789 Bochum kaelicke@googlemail.com 